\title{
Joseph Grange, 1997, Nature, an environmental cosmology
}

State University of New York Press, Albany, New York, 272 p.

Philippe Vaillant

\section{(2) OpenEdition}

\section{Journals}

Édition électronique

URL : http://journals.openedition.org/rge/1915

DOI : $10.4000 /$ rge. 1915

ISSN : 2108-6478

Éditeur

Association des géographes de l'Est

Édition imprimée

Date de publication : 1 janvier 2009

ISSN : 0035-3213

\section{Référence électronique}

Philippe Vaillant, « Joseph Grange, 1997, Nature, an environmental cosmology », Revue Géographique de l'Est [En ligne], vol. 49 / 2-3 | 2009, mis en ligne le 06 octobre 2010, consulté le 08 septembre 2020. URL : http://journals.openedition.org/rge/1915; DOI : https://doi.org/10.4000/rge.1915

Ce document a été généré automatiquement le 8 septembre 2020

Tous droits réservés 


\title{
Joseph Grange, 1997, Nature, an environmental cosmology
}

State University of New York Press, Albany, New York, 272 p.

\author{
Philippe Vaillant
}

$1 \quad$ S'il est présenté dans une revue de géographie, c'est que cet ouvrage tente d'être «passeur de frontières» entre des disciplines peu habituées à cohabiter. Il est l'application de l'approche whiteheadienne à l'environnement naturel et urbain. L'approche concernant l'environnement naturel est l'objet de la présentation faite ciaprès, celle concernant l'environnement urbain est traitée dans un deuxième ouvrage paru en 1999 et qui reste à être traduit. Dans ses deux textes, J. Grange a voulu présenter une approche cosmologique commune pour la Nature et la Cité. Il propose ainsi une sortie du dualisme classique en faveur du schème organique whiteheadien exposé dans l'ouvrage de référence d'A.N.Whitehead Procès et réalité, un essai de cosmologie, traduit chez Gallimard en 1995 (579 p).

2 L'ouvrage analysé est composé de trois parties équilibrées, d'environ 80 pages chacune, portant sur le schème cosmologique, la nature et la vision éthique. Son écriture relève d'un effort spéculatif pour comprendre de façon systématique les réalités entrelacées de nos environnements contemporains. Il propose de sortir du matérialisme scientifique hérité de Descartes et Hume qui sépare le fait de la valeur, pour passer à une cosmologie où "être" est toujours "être une valeur" (p.16) et où exister est la réalisation d'une valeur, à la suite de Whitehead, de Peirce. Chaque valeur réalisée est une intégrité environnementale. Chaque environnement est un "Unisson de devenir". Pour J. Grange, cette dernière notion est un processus, et non pas un état de l'être, et par conséquent l'unité fondamentale indique la présence réelle de relations internes dans le venir à l'être et la disparition des êtres environnementaux (p.19). Dans cette approche, la catégorie ultime est la créativité, source de nouveauté sans fin, qui marque nos régions naturelles et urbaines. Elle est ce que les chinois appellent le Tao. Ainsi, l'approche évoquée tente de tracer un pont entre Occident et Orient.

Pour dépasser le dogme moderne d'une science basée sur la perception sensible, l'ouvrage invite de passer à la notion de «sentirs» (feelings) physiques, conceptuels, 
propositionnels ou de jugement. Il détaille à la suite les critères de scientificité du schème (la cohérence, la logique du vague, la nécessité -critère internes- puis l'adéquation et l'applicabilité -critères externes-). La logique du vague ne signifie pas, ici, ambigu, flou ou manquant de définition formelle. Il revient au grand génie du philosophe américain Charles S. Peirce d'avoir reconnu la qualité logique du vague invoquée pour compléter Whitehead. J. Grange décrit ensuite les 4 catégories de base de l'analyse environnementale que sont l'eccéité (principe qui fait qu'une essence est rendue individuelle), le contraste, la configuration et la transmission.

4 Fort de la clarté des notions mises en oeuvre, l'auteur, dans la deuxième partie, montre comment ces catégories permettent de rendre compte du temps, de l'espace, du phénomène de la Vie et de la Nature.

5 La troisième partie est consacrée à la formulation de l'éthique environnementale qui en découle. Dans un premier temps, l'auteur fait le lien avec les approches traditionnelles, et celles formulées par Kohack ou H. Rolston afin de bien dégager ensuite les traits novateurs de l'éthique environnementale organique.

6 La traduction attendue du second tome de la pensée de Grange doit contribuer à établir les fondations d'une approche organique whiteheadienne et peircienne des paysages naturel et urbain. L'élément méthodologique fort de cette démarche est la logique $d u$ vague, à bien distinguer d'une logique du flou. 